\section{Focal Cemento-Osseous Dysplasia: A Case Report}

\section{Genno Karam N1, Bou Tayeh Rima², Naserdine Sawsan ${ }^{3}$, Nasseh $\mathrm{I}^{1}$ and Zeinoun $\mathrm{T}^{2 *}$}

${ }^{1}$ Department of Oral and Maxillofacial Radiology, Dental school, Lebanese University, Beirut, Lebanon

${ }^{2}$ Department of Oral and Maxillofacial Surgery, Dental school, Campus Hadath, Lebanese University, Beirut, Lebanon

${ }^{3}$ Department of public health, Dental school, Lebanese University, Beirut, Lebanon

\begin{abstract}
Cemento-Osseous Dysplasia (COD) is the most common osseous fibrous lesion, which involves replacement of the normal bone with a poorly cellularized cementum-like material and cellular fibrous connective tissue [1]. It can be observed in the periapical area of vital teeth or in extraction sites. The radiographic features are variable, comprising a combination of radiolucent and radiopaque patterns. This lesion is classified into three subtypes according to its clinical and radiographic features: periapical, focal, and florid [2-3]. This report describes a case of a 30 year old Lebanese woman who was diagnosed with focal cemento-osseous dysplasia FocCOD on the base of clinical and radiographic findings.
\end{abstract}

\section{Introduction}

The high incidence and broad spectrum of conditions causing periapical radiolucencies make it imperative that all dental clinicians acquire a broad and comprehensive working knowledge. Histologically, COD appears to affect tooth-bearing areas probably derived from the periodontal ligament and from the cementum or cemental-like tissues [4]. Focal Cemento-Osseous Dysplasia (FocCOD) is an asymptomatic condition usually observed incidentally on routine radiographs. The etiology and pathogenesis is unknown, and this lesion is considered to be a reactive or a dysplastic process of periapical region. Usually, it affects two or more mandibular anterior teeth, and the radiographic appearance varies depending on the state of development. In rare cases, the lesion may affect only one tooth and thus mimics an apical granuloma or cyst [5].

Since fibro-osseous lesions tend to have a wide range of presenting clinical and radiographic appearances, the aim of this case report is

*Corresponding author: Toni Zeinoun, Department of Oral and Maxillofacial Surgery, Dental school, Campus Hadath, Lebanese University, Beirut, Lebanon, Tel: +961 3555566; E-mail: zeinountoni@gmail.com

Citation: Karam NG, Rima BT, Sawsan N, Nasseh I and Zeinoun T (2014) Focal Cemento-Osseous Dysplasia: A Case Report. J Clin Stud Med Case Rep 1: 003.

Received: August 21, 2014; Accepted: November 20, 2014; Published: December 04, 2014 to show that it is imperative for the dentist to be familiar with the different presentations of these benign lesions in order to establish an appropriate diagnosis and treatment planning.

\section{Case Report}

A 30-year-old Lebanese female patient (Figure 1) presented at Lebanese University, School of Dentistry, Beirut, Lebanon for dental care. The patient was healthy and her physical examination showed no abnormality. She had no history of mandibular trauma. On extra-oral examination, no abnormalities were observed. Intraoral examination revealed normal oral mucosa and absence of soft tissue expansion. Panoramic radiograph showed an asymptomatic unilocular radiolucent-radiopaque mixed lesion associated with the apex of the right mandibular lateral incisor (Figure 2).
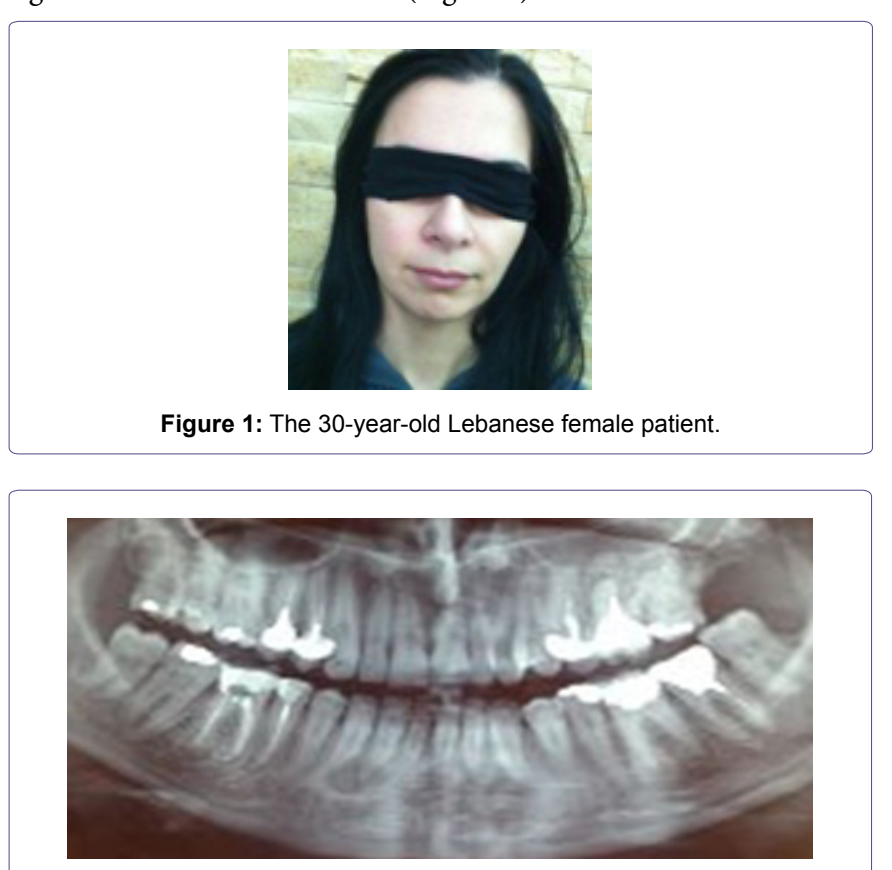

Figure 2: A panoramic view demonstrates the extent of the lesion.

Periapical radiograph revealed a mixed density lesion associated with the apex of the tooth 42 , with distinct borders and a somewhat unilocular appearance. The bone adjoining the borders was sclerosed in several locations, giving a pseudocortical appearance. There was no root resorption or tooth displacement. The lamina dura surrounding apical areas of the involved tooth was lost. Periodontal ligament space widening was found around the root (Figure 3).

By using a CBCT, the state of the lesion relative to the buccal and lingual cortical plates could be assessed, which might not be possible on the conventional radiographs. $\mathrm{CBCT}$ axial cuts showed the bucco-lingual extent of the lesion with erosion of lingual cortical plate (Figure 4).

Para-axial CBCT cuts revealed the relationship of the lesion with the third apical part of the root of 42. Also note the well-demarcated radiopaque mass in the centre of the lesion (Figure 5). 


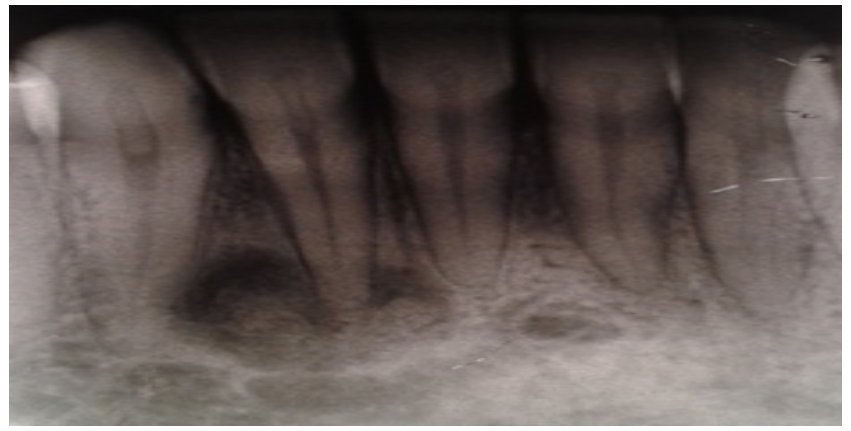

Figure 3: Periapical radiograph revealed a mixed density lesion associated with the apex of teeth 42 .

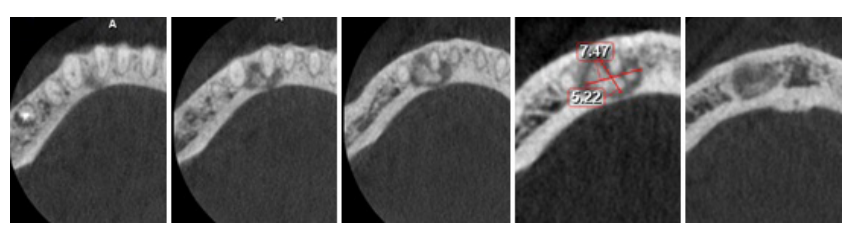

Figure 4: $C B C T$ axial cuts showing the bucco-lingual extent of the lesion with erosion of lingual cortical. The lesion measured approximately $5.22 \mathrm{~mm} \times 7.47$ $\mathrm{mm}$.

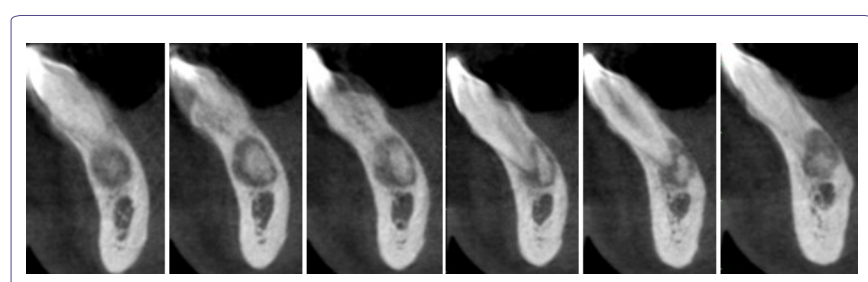

Figure 5: Note the intimate relationship of the lesion with the $1 / 3$ apical part of root of 42 .

Dental pulp tests are investigations that provide valuable diagnosis and treatment planning. They include thermal and electric tests, which extrapolate pulp health from sensory response.The involved tooth (42) was vital in an electric stimulation test as in thermal test.

We decided to abstain from surgical intervention and have kept the patient under regular clinico-radiological follow-up for two years (Figures 6 and 7)
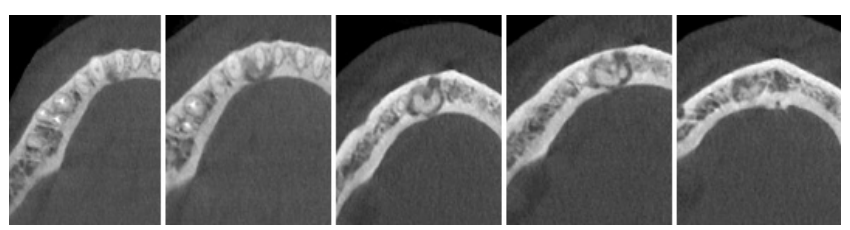

Figure 6: CBCT axial cuts at a 2-year follow up, showing no modifications of the lesion.

\section{Discussion}

Benign fibro-osseous lesions are disturbances in bone metabolism where normal bone is replaced by a connective tissue matrix. Lesions in this category include fibrous dysplasia, focal and florid cemento-osseous dysplasias as well as periapical cemental

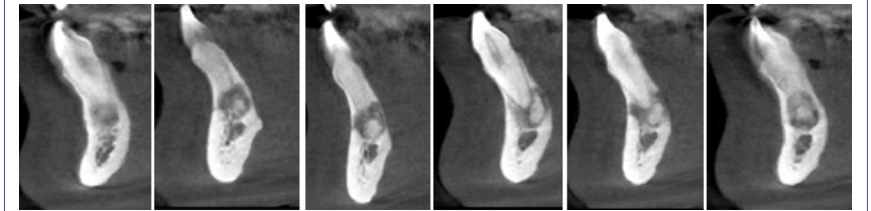

Figure 7: СВCT para-axial cuts at a 2-year follow up, showing no modifications of the lesion.

dysplasia. Cemento-ossifying fibroma, although classified as and behaving like a benign neoplasm, is often grouped with the fibro-osseous lesions due to comparable histology [6]

The fibro-osseous lesions have many common characteristics including clinical, radiographic and microscopic features, causing considerable diagnostic confusion [7]. In fact, the histopathological appearances of all fibro-osseous lesions are very similar, if not identical, making diagnosis difficult based on microscopic features alone [8]. Waldron commented further that "in the absence of good clinical and radiologic information, a pathologist can only state that a given biopsy is consistent with a fibro-osseous lesion" and that "with adequate clinical and radiologic information, most lesions can be assigned with reasonable certainly into one of several categories" [9]. The differential diagnosis should consider the stage of development of the lesion and include periapical granuloma or cyst and chronic osteomyelitis at osteolytic stage, whereas in the mixed and radio opaque stages, like our case, chronic sclerosing osteomyelitis, ossifying/cementifying fibroma, cementoblastoma should be considered $[8,9]$.

Paget's disease remains as a differential diagnosis for COD and has to be ruled out as the former may transform into osteosarcoma. FocCOD is centered above the inferior alveolar canal whereas Paget's involve the entire mandible. Also the dysplastic lesion of Paget's disease is always polyostotic and shows an increase in the serum alkaline phosphatase level [10].

The second most common lesion that interferes with the diagnosis of COD is the monostotic fibrous dysplasia. Clinically, the maxilla is affected more often than the mandible and lesions are first detected in the late first and early second decades [6].

The present case may resemble chronic sclerosing osteomyelitis but there is no clinical history of pain or pus drainage in relation to the lesion site [6]. So we ruled out such differential diagnosis.

The present case shows the cemento-osseous tissue with absence of fusion to the apex of the tooth root, which rules out the possibility of cementoblastoma [11].

It is important to differentiate lesions of $\mathrm{COD}$ from a true neoplasm, Ossifying Fibroma (OF). A periodic follow-up is recommended in order to ensure that no further enlargement or expansion occurs, features that argue in favor of an ossifying fibroma that was merely encountered in an early stage of development [6]. This differential diagnosis is also ruled out because in our case, at a 2-year follow-up, the lesion didn't encounter any modification (Figure 6).

The classification of cemento-osseous lesions of the jaws released by WHO in 1992 is based on age, sex, histopathologic, radiographic and clinical characteristics as well as location of lesion [12]. The three entities (focal, periapical, florid) have differences only in clinical and radiological presentation [4]. The term osseous dysplasia has been 
re-introduced in the new classification of head and neck tumors given by WHO (July 2005), thus pooling the different entities under one heading [13].

FocCOD was first suggested by Summerlin and Tomich, primarily according to the location of dysplastic areas of the bone. When the lesion is in an edentulous site, not associated with a tooth apex, the term "Focal Cemento-Osseous Dysplasia" is used [14]. Periapical Cemento-Osseous Dysplasia (PCOD) and Focal Cemento-Osseous Dysplasia (FocCOD) are two different terms for the same reactive lesion. Florid cemento-osseous dysplasia (Fl.COD) denotes an extensive process with multifocal involvement of the jaws by lesional tissue with the same microscopic appearance as is encountered with PCOD and FocCOD [6].

FocCOD is usually found during routine radiographic examination. Although it is generally accepted that symptomless FocCODs need no treatment, their clinical importance has been emphasized by their presence in edentulous sites required for osseointegrated implants [15]. In seventy percent of the cases, it displays an intimate relationship to the periapex of teeth with vital pulps, as in our case. The remaining $21 \%$ is found in the sites of previous extraction and may mimic a residual cyst. Local jaw expansion and mild discomfort may be reported in about one-third of these patients $[5,16,17]$.

FocCOD has been described as having three developmental stages, each with specific radiographic features. In the early or osteolytic stage, radiographs show a well-defined radiolucent lesion. In the intermediate stage, it displays a mixture of radiolucent and radio opaque architecture. The last mature stage is characterized by a definite radiopacity, present in the major part of the lesion [15]. In our case, the radiograph showed a mixed density lesion with distinct borders.

Periapical Cemento-Osseous Dysplasia (PCOD) is a subtype of cemento-osseous dysplasia that usually occurs in middle-aged black women [18]. It is unusual for a PCOD to produce a detectable expansion of the cortical plate [19].

The word florid introduced by Melrose et al. in 1976 is a disease with multiple bilateral and often symmetrically extensive lesions throughout the maxillofacial region. Lesions are often asymptomatic and indolent in nature and may present as incidental findings on radiographs [20]. Radiographically, the lesions present a mixed radiolucent and radiopaque "cotton wool" appearance [21].

Jaw expansion is not often reported, as in our case at the 2-year follow up [19]. In contrast, Loh and Yeo [22] and Tonioli and Schindler [23] reported a considerable expansion based on their observations of clinical examination alone. Melrose et al., [24] also reported jaw expansion with intact thinned cortices in their series of 34 florid COD.

In some cases, a familial trend has been observed [25]. The process may be totally asymptomatic and, in such cases, the lesion is detected on radiographs for some other purposes [26]. Symptoms such as dull pain or drainage are almost always associated with exposure of sclerotic calcified masses in the oral cavity. This may occur as the result of progressive alveolar atrophy under a denture or after extraction of teeth in the affected area [27].

The diagnosis of our case is established radiographically. Biopsy is not indicated, not only for the embodiment of the characteristic features leading to accurate diagnosis but also because several authors have reported that following extraction of tooth in FocCOD, the patients presented with poor socket healing and even sequestrum formation, thus complicating the condition [10].

The management of COD is conservative unless when symptomatic as a result of superimposed infection as COD is avascular and therefore susceptible to infection. Therefore, when the lesion becomes exposed into the oral cavity for example following tooth extraction, surgical biopsy or erosion of the overlying mucosa infection can occur. Osteomyelitis resulting from infected lesions is difficult to treat as there is widespread sclerosis with consequent poor blood supply. In some rare situations where COD has caused an aesthetically unpleasant swelling, a surgical intervention may be required [28]. Asymptomatic patients of FocCOD generally do not require any treatment and should be kept under observation, and regular radiological follow-up [15].

\section{Conclusion}

The diagnostic confusion often associated with COD has led to mismanagement, often rendering these cases problematic [29]. Therefore, the role of the oral and maxillofacial radiologist in the differential diagnosis is an important and essential one [30]. Prognosis is excellent, but follow-up is warranted because of the possibility of progression to florid COD [31].

\section{References}

1. Huh JK, Shin SJ (2013) Misdiagnosis of florid cemento-osseous dysplasia leading to unnecessary root canal treatment: a case report. Restor Dent Endod 38: 160-166.

2. Dağistan S, Tozoğlu U, Göregen M, Cakur B (2007) Florid cemento-osseous dysplasia: a case report. Med Oral Patol Oral Cir Bucal 12: E348-350.

3. Komabayashi T, Zhu Q (2011) Cemento-osseous dysplasia in an elderly Asian male: a case report. J Oral Sci 53: 117-120.

4. Paul M Speight, Roman Carlos (2006) Maxillofacial fibro osseous lesions. Current Diagnostic Pathology 12: 1-10.

5. Bhandari R, Sandhu SV, Bansal H, Behl R, Bhullar RK (2012) Focal cemento-osseous dysplasia masquerading as a residual cyst. Contemp Clin Dent 3: $60-62$.

6. Eversole R, Su L, El Mofty S (2008) Benign fibro-osseous lesions of the craniofacial complex. A review. Head Neck Pathol 2: 177-202.

7. M Mupparapu, SR Singer, Milles M, Rinaggio J (2005) Simultaneous presentation of focal cemento-osseous dysplasia and simple bone cyst of the mandible masquerading as multilocular radiolucency. Dentomaxillofacial Radiology 34: 39-43.

8. Drazić R, Minić AJ (1999) Focal cemento-osseous dysplasia in the maxilla mimicking periapical granuloma. Oral Surg Oral Med Oral Pathol Oral Radiol Endod 88: 87-89.

9. Waldron CA (1993) Fibro-osseous lesions of the jaws. J Oral Maxillofac Surg 51: 828-835.

10. Young SK, Markowitz NR, Sullivan S, Seale TW, Hirschi R (1989) Familial gigantiform cementoma: classification and presentation of a large pedigree. Oral Surg Oral Med Oral Pathol 68: 740-747.

11. Napier Souza L, Monteiro Lima Júnior S, Garcia Santos Pimenta FJ, Rodrigues Antunes Souza AC, Santiago Gomez R (2004) Atypical hypercementosis versus cementoblastoma. Dentomaxillofac Radiol 33: 267-270.

12. Srivastava A, Agarwal R, Soni R, Sachan A, Shivakumar GC, et al. (2012) Familial florid cemento-osseous dysplasia: a rare manifestation in an Indian family. Case Rep Dent 2012: 574125. 
13. Barnes L, Eveson JW, Reichart P, Sidransky D, (2005) Head and Neck Tumors. Lyon: IARC Press; 2005. World Health Organization Classification of Tumors. Pathology and Genetics.

14. Galgano C, Samson J, Küffer R, Lombardi T (2003) Focal cemento-osseous dysplasia involving a mandibular lateral incisor. Int Endod J 36: 907-911.

15. Macdonald-Jankowski DS (2008) Focal cemento-osseous dysplasia: a systematic review. Dentomaxillofac Radiol 37: 350-360.

16. Alsufyani NA, Lam EW (2011) Osseous (cemento-osseous) dysplasia of the jaws: clinical and radiographic analysis. J Can Dent Assoc 77: 70.

17. Galgano C, Samson J, Küffer R, Lombardi T (2003) Focal cemento-osseous dysplasia involving a mandibular lateral incisor. Int Endod J 36: 907-911.

18. Eskandarloo A, Yousefi F (2013) CBCT findings of periapical cemento-osseous dysplasia: A case report. Imaging Sci Dent 43: 215-218.

19. Kawai T, Hiranuma H, Kishino M, Jikko A, Sakuda M (1999) Cemento-osseous dysplasia of the jaws in 54 Japanese patients: a radiographic study. Ora Surg Oral Med Oral Pathol Oral Radiol Endod 87: 107-114.

20. Smith MH, Harms PW, Newton DW, Lebar B, Edwards SP, et al. (2011) Mandibular Actinomyces osteomyelitis complicating florid cemento-osseous dysplasia: case report. BMC Oral Health 11: 21.

21. Onder B, Kurșun S, Oztaș B, Barıș E, Erdem E, (2013) Florid osseous dysplasia in a middle-aged Turkish woman: A case report. Imaging Sci Dent 43 : 197-200.

22. Loh FC, Yeo JF (1989) Florid osseous dysplasia in Orientals. Oral Surg Oral Med Oral Pathol 68: 748-753.
23. Tonioli MB, Schindler WG (2004) Treatment of a maxillary molar in a patient presenting with florid cemento-osseous dysplasia: a case report. J Endod 30: $665-667$.

24. Melrose RJ, Abrams AM, Mills BG (1976) Florid osseous dysplasia. A clinical-pathologic study of thirty-four cases. Oral Surg Oral Med Oral Pathol 41: 62-82.

25. Toffanin A, Benetti R, Manconi R (2000) Familial florid cemento-osseous dysplasia: a case report. J Oral Maxillofac Surg 58: 1440-1446.

26. Gariba-Silva R, Sousa-Neto MD, Carvalho JR Jr, Saquy PC, Pecora JD (1999) Periapical cemental dysplasia: case report. Braz Dent J 10: 55-57.

27. Said-al-Naief NA, Surwillo E (1999) Florid osseous dysplasia of the mandible: report of a case. Compend Contin Educ Dent 20: 1017-1019, 1022-1028.

28. Olusanya AA, Adeyemi BF, Adisa AO (2012) Concurrent cemento-osseous dysplasia and osteogenic sarcoma: report of two cases. Case Rep Med 2012: 180561.

29. Islam MN, Cohen DM, Kanter KG, Stewart CM, Katz J, et al. (2008) Florid cemento-osseous dysplasia mimicking multiple periapical pathology--an endodontic dilemma. Gen Dent 56: 559-562.

30. Alsufyani NA, Lam EW (2011) Cemento-osseous dysplasia of the jaw bones: key radiographic features. Dentomaxillofac Radiol 40: 141-146.

31. Summerlin DJ, Tomich CE (1994) Focal cemento-osseous dysplasia: a clinicopathologic study of 221 cases. Oral Surg Oral Med Oral Pathol 78: 611620. 